\title{
Beratung in der Pflege - der Wittener Werkzeugkasten
}

\author{
Andreas Kocks
}

Online publiziert: 30. November 2011

(C) Springer-Verlag 2011

Pflege ist Begegnung. Jede Pflegesituation ist durch Interaktion und Kommunikation gekennzeichnet. Der Anteil der Beratungsgespräche mit Patienten und Angehörigen ist von großer Bedeutung. Sie fordern fundierte, auf ihre individuelle Situation hin angepasste Beratung, Informationen und Anleitungen ein. Pflegende weisen als größte Berufsgruppe im Gesundheitswesen die größte Nähe zum Patienten auf. Sie sind bei Fragen vielfach die ersten und nicht selten auch die einzigen Ansprechpartner. Durch eine Kommunikation auf Augenhöhe und hohe Vertrauensstellung sind es gerade sie, denen Fragen zur Integration von Krankheit, Therapie und Gesundheitseinbußen in einen gelebten Alltag gestellt werden.

In Pflegeausbildung und Aussagen zum Beruf spielt Beratung eine herausragende Rolle. Dies spiegelt sich in der eigenen Reflexion der Pflegepraxis nur ansatzweise wieder. Vielfach werden kommunikative Handlungen unterbewertet, passieren beiläufig, handlungsbegleitend, ungeplant und entziehen sich durch fehlende Dokumentation der interprofessionellen Anschlussfähigkeit.

Die studentische Arbeitsgruppe Patientenedukation von Angelika Abt-Zegelin hat sich mit dem Diplompsychologen G. G. Bamberger mit professioneller Beratung in der Pflege auseinandergesetzt. Ausgangspunkt bildete die phänomenologische Betrachtung pflegerischen Verhaltens in Beratungsgesprächen. Mit sehen, hören, fühlen, sprechen und tun wurden fünf Beratungsmodalitäten identifiziert.
A. Kocks, MSc $(\bowtie)$

Department Pflegewissenschaft, Universität Witten/Herdecke,

Witten, Deutschland
Wie diese Modalitäten im Sinne einer hilfreichen Begegnung hergestellt werden bildete den Focus der weiterführenden Fragestellungen. Ziel war es, pragmatische, handlungsleitende Beratungswerkzeuge (Tools) zu finden. Mit Achtsamkeit, Einlassung, Empathie, Resourcing und Berührung wurden Werkzeuge gefunden, die den Patienten in den Blick nehmen. Soll Beratung gelingen, ist es darüber hinaus wichtig, dass der Berater seine eigene Position reflektiert. Introspektion, Intuition, Selbst-Spürung, Selbstverständnis und Grounding sind Werkzeuge, die diesen Prozess unterstützen. Vor dem Hintergrund der Leistungsverdichtung in der Pflege und dem Umgang mit der eigenen Gesundheit stellen die „Wittener Werkzeuge“ eine innovative Erweiterung pflegerischer Beratungskonzepte dar. 